\title{
Novel Mutations in Cholangiocarcinoma with Low Frequencies Revealed by Whole Mitochondrial Genome Sequencing
}

\author{
Kanha Muisuk $^{1,2,3}$, Atit Silsirivanit ${ }^{1,3}$, Kanokwan Imtawil $^{1}$, Suphawadee Bunthot ${ }^{1}$, \\ Ake Pukhem $^{3,4}$, Chawalit Pairojkul ${ }^{3,5}$, Sopit Wongkham ${ }^{1,3}$, Chaisiri Wongkham ${ }^{1,3 *}$
}

\begin{abstract}
Background: Mitochondrial DNA (mtDNA) mutations have been shown to be associated with cancer. This study explored whether mtDNA mutations enhance cholangiocarcinoma (CCA) development in individuals. Materials and Methods: The whole mitochondrial genome sequences of $25 \mathrm{CCA}$ patient tissues were determined and compared to those of white blood cells from the corresponding individuals and 12 healthy controls. The mitochondrial genome was amplified using primers from Mitoseq and compared with the Cambridge Reference Sequence. Results: A total of 161 mutations were identified in CCA tissues and the corresponding white blood cells, indicating germline origins. Sixty-five $(\mathbf{4 0} \%)$ were new. Nine mutations, representing those most frequently observed in CCA were tested on the larger cohort of 60 CCA patients and 55 controls. Similar occurrence frequencies were observed in both groups. Conclusions: While the correspondence between the cancer and mitochondrial genome mutation was low, it is of interest to explore the functions of the missense mutations in a larger cohort, given the possibility of targeting mitochondria for cancer markers and therapy in the future.
\end{abstract}

Keywords: Biliary cancer - bile duct cancer - mitochondrial mutation - mitochondrial genome

Asian Pac J Cancer Prev, 16 (5), 1737-1742

\section{Introduction}

Mitochondria are the organelles that play many important roles including the generation of ATP, production of reactive oxygen species (ROS), and initiation and execution of apoptosis (Kim et al., 2006, Fogg et al., 2011). The human mitochondrial DNA (mtDNA) contains 37 genes that express 13 messenger RNA (mRNA), two ribosomal RNAs (rRNA) and 22 transfer RNAs (tRNA) (Anderson et al., 1981). The 13 polypeptides products make up the subunits of several enzyme complexes necessary for respiratory and oxidative phosphorylation (DiMauro and Schon, 2003). Although the number is small, these polypeptides are essential for the proper assembly and activity of a large host of other enzyme complexes (Holt et al., 1990; Bai and Attardi, 1998; Radpour et al., 2009).

Cancer is considered to be a heterogeneous set of diverging molecular and cellular features. There has been an accumulation of evidences suggesting that the mitochondria plays a key role in cancer (reviewed in Chatterjee et al., 2006; Gaude and Frezza, 2014). Mutations to mitochondrial DNA may lead to mitochondrial dysfunction and the metabolic reprogramming of cancer cells leading to the modulation in cellular processes involved in the initiation and progression of cancer. Several somatic and germline mtDNA mutations have been identified in the coding and non-coding regions in various types of human cancers (reviewed in Chatterjee et al., 2006, Brandon et al., 2006). Recently, whole genome analysis of mtDNA also revealed mutations in various cancers including breast cancer, colorectal cancer, gastric cancer and hepatocellular carcinoma (Ma et al., 2010; Hung et al., 2010; Yin et al., 2010).

Cholangiocarcinoma (CCA) is rare worldwide but the incidence is high in the Northeast Thailand. Chronic inflammation of biliary tract is known to be the underlined the pathogenesis of CCA. A meta-analysis of population based studies showed that patients with inflammatory bowel diseases e.g., Crohn's disease and ulcerative colitis, had an increased risk of CCA (Huai et al., 2014). The etiological and epidemiological studies of CCA in Thailand link CCA to parasitic infections of the liver flukes Opisthorchis viverrini (OV) [reviewed in (Sripa and Pairojkul, 2008)]. However, only 0.5-1\% of OV-infected individuals develop CCA (Mairiang et al., 2006). The results suggest other factors that promote patho- and tumorigenesis in OV-infected individuals. Recently, the polymorphisms of IL-6 receptor (Prayong et al., 2014) and the NRF2 rs6726395 (Khunluck et al.,

${ }^{1}$ Department of Biochemistry, ${ }^{2}$ Department of Forensic Medicine, ${ }^{3}$ Liver Fluke and Cholangiocarcinoma Research Center, ${ }^{4}$ Department of Surgery, ${ }^{5}$ Department of Pathology, Faculty of Medicine, Khon Kaen University, Khon Kaen, Thailand *For correspondence: chaisiri@kku.ac.th 


\section{Kanha Muisuk et al}

2014) were shown to be related to OV-associated CCA. However, the mitochondrial mutation in CCA has, to our best knowledge, not been studied.

In the present study, we determined the association between mtDNA mutations and carcinogenesis and pathogenesis of CCA. Whole mitochondrial genome of CCA patient tissues and corresponding white blood cells were sequenced and compared to the white blood cells from healthy controls. Several new mtDNA mutations found only in CCA patients were addressed.

\section{Materials and Methods}

\section{Tissues and white blood cells}

Formalin fixed paraffin embedding (FFPE) tissues $(n=25)$ and white blood cells (WBC) of CCA patients $(n=60)$ were obtained from the biobank of Liver Fluke and Cholangiocarcinoma Research Center, Faculty of Medicine, Khon Kaen University, Khon Kaen, Thailand. All CCA tissues were histologically verified. Blood were collected from healthy subjects $(n=55)$ who were Northeastern Thai residences with written informed consent. The ethic committee for human research of Khon Kaen University approved the protocol of study.

\section{DNA extraction}

CCA cells from the FFPE tissue slides were located and micro-dissected. At least 1,000 cells were collected for DNA extraction. Dissected CCA cells, (with RNAasetreated) by the QIAampFFPE tissue DNA Micro kit (QIAGEN, Germany) according to the instruction of the manufacturer. The extracted DNA were kept in acetateEDTA buffer at $-20^{\circ} \mathrm{C}$ until analysis.

\section{Amplification reaction and amplicon cleaned-up}

The complete human mitochondrial genomes were amplified (singleplex reactions, mitoSEQr ${ }^{\mathrm{TM}}$ ) with 46 pairs of primers which generated 46 overlapping fragments covering the whole mitochondrial genome. Amplification reactions were carried out on a GeneAmp 9700 Thermal cycler (ABI) according to the standard protocol. All amplicons gaving positive results were cleaned-up to remove excess primers and dNTPs with 2 $\mu 1$ of ExoSAP-IT (USB Corporation) at $37^{\circ} \mathrm{C}$ for $30 \mathrm{~min}$ and heat inactivation at $80^{\circ} \mathrm{C}$ for $15 \mathrm{~min}$, and kept on ice immediately.

\section{The sequencing reaction and automate sequencing run}

Both forward and reverse sequencing were carried out using BigDye Terminator V3.1 cycle sequencing reaction kit (Applied Biosystems, USA) according to the standard protocol. Sequencing reaction products were purified from residual dye terminator using EDTA/ethanol precipitation. The final residue was resuspended with $12 \mu 1$ Hi-Di formamide for electrophoresis. Electrophoresis was performed on the Genetic Analyzer ABI 3130 (Applied Biosystems, USA) using POP6 and a $36 \mathrm{~cm}$ array. The run module conditions were as follows: oven temperature: $60^{\circ} \mathrm{C}$, injection polymer $20 \mu \mathrm{l} / \mathrm{cap}$, electrophoresis (EP) voltage: $15 \mathrm{kV}$, EP current: $34 \mu \mathrm{A}$, and Laser power: 15 $\mathrm{mW}$.

\section{Data analysis of mtDNA mutations}

All mtDNA sequencing data were assembled using SeqScape ${ }^{\circledR}$ software v2.6 (Applied Biosystem, USA), and mtDNA mutations were identified using Cambridge Reference Sequence (MITOMAP). Sequencing reactions were repeated for any reaction with unassembled sequencing data.

\section{Statistical analysis}

Statistical calculation was performed using SPSS 16.0 Windows Evaluation software (SPSS Inc. Chicago, IL). The Chi square $\left(\mathrm{x}^{2}\right)$-test was used for comparison between two groups. Statistical significance was established at $p<0.05$.

\section{Results}

There were 60 CCA patients ( 38 males and 22 females, age ranged $44-76$ years) and 55 healthy controls ( 30 males and 25 females, age ranged 19-73 years) included in this study. Whole mtDNA sequencings were performed on a pilot set of 25 CCA patient tissues and paired white blood cells. Similar sequencing was also performed in white blood cells of 12 controls.

All mtDNA mutations identified in CCA patient tissues were substitution of single point mutations and germline origins

A total of 161 mutations were found in CCA tissues ranging from 6-25 mutations per individual (Figure 1A). All mutations were substitutions of single points corresponding to 7 transversions $(4.3 \%)$ and 154 transitions (95.6\%). Of 161 mutations, 103 were silent $(64 \%)$ and 58 were missense $(36 \%)$ (Table 1). The most frequent mutation found in the 13 mitochondria encoded genes was on ND5 of complex I, and the least frequent mutation was on ATP8.

To investigate whether the mtDNA mutation found in CCA was of somatic or germline origins, the whole mitochondrial genomes of the matched white blood cells were determined and analyzed in a similar manner. Of 25 CCA cases, the mutation patterns observed in individual tumor tissue and white blood cells were identical (data not shown). This result confirms the germline origin of the mutations in the CCA tissues.

Numerous new mutations were determined in mtDNA from CCA patients

The association of the mtDNA mutations found in CCA patients with the disease was further explored. To this end, whole mitochondrial genome of white blood cells from 12 healthy persons were analyzed. There were 101 mutations with between 8 and 23 mutations per individual (Figure 1B). Of the total 74 were silent (73\%) and 27 were missense $(27 \%)$. The mutations detected in CCA patients and healthy subjects were compared (Figure 2). Of the total 262 mutations, 61 mutations were commonly found in both groups, 100 mutations were found only in CCA patients and 40 mutations were found only in healthy persons (Figure 2A). Of the 161 mutations found in CCA patients, $96(60 \%)$ mutations were previously reported, 
Table 1. Number of Total, Silent and Missense Mutations found in Mitochondrial Genes

\begin{tabular}{|c|c|c|c|c|c|c|}
\hline \multicolumn{4}{|c|}{ mt DNA geneCCA patients $(n=25)$} & \multicolumn{3}{|c|}{ Healthy subjects $(n=12)$} \\
\hline & Total & silent & missense & Total & silent & missense \\
\hline \multicolumn{7}{|c|}{ Complex I } \\
\hline ND1 & 18 & 10 & 8 & 10 & 7 & 3 \\
\hline ND2 & 11 & 8 & 3 & 10 & 7 & 3 \\
\hline ND3 & 9 & 6 & 3 & 8 & 7 & 1 \\
\hline ND4 & 6 & 5 & 1 & 7 & 5 & 2 \\
\hline ND4L & 4 & 2 & 2 & 0 & 0 & 0 \\
\hline ND5 & 25 & 13 & 12 & 18 & 9 & 9 \\
\hline ND6 & 8 & 0 & 8 & 4 & 1 & 3 \\
\hline \multicolumn{7}{|c|}{ Complex III } \\
\hline Cyt b & 19 & 13 & 6 & 9 & 8 & 1 \\
\hline \multicolumn{7}{|c|}{ Complex IV } \\
\hline $\mathrm{CO} \mathrm{I}$ & 17 & 16 & 1 & 13 & 13 & 0 \\
\hline $\mathrm{CO}$ II & 15 & 12 & 3 & 9 & 8 & 1 \\
\hline CO III & 10 & 9 & 1 & 7 & 6 & 1 \\
\hline \multicolumn{7}{|c|}{ Complex V } \\
\hline ATP6 & 16 & 7 & 9 & 6 & 3 & 3 \\
\hline ATP8 & 3 & 2 & 1 & 0 & 0 & 0 \\
\hline Total & 161 & 103 & 58 & 101 & 74 & 27 \\
\hline$\%$ & 100 & 64 & 36 & 100 & 73.3 & 26.7 \\
\hline
\end{tabular}

$1 \mathrm{~A}$

- Total mutation $\quad$ Silent mutation $\|$ Missense mutation

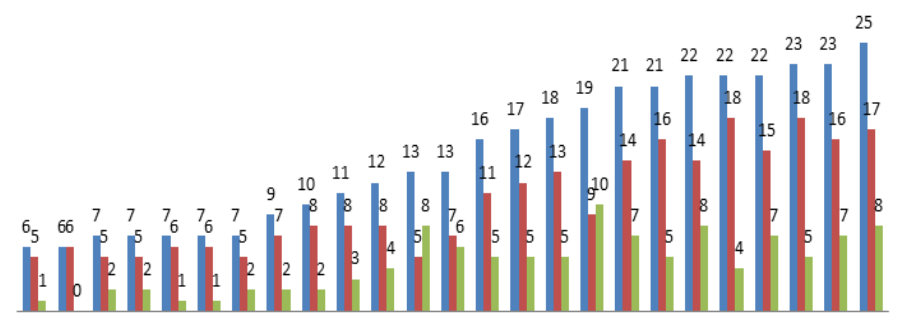

$1 \mathrm{~B}$

\begin{tabular}{llllr}
\hline mt gene & NT position & AA change & \multicolumn{2}{c}{ CCA } \\
\cline { 4 - 5 } & & & $\mathrm{n}=25$ & $\%$ \\
\hline \multicolumn{2}{c}{ Previously reported } & & & \\
ND4L & T10609C & I $\rightarrow$ T & 2 & 8 \\
Cyt b & G15346A & silent & 4 & 16 \\
ND2 & A5351G & silent & 3 & 12 \\
ND2 & T5465C & silent & 2 & 8 \\
ND3 & T10238C & silent & 2 & 8 \\
ND5 & G12630A & silent & 2 & 8 \\
CO III & T9716C & silent & 2 & 8 \\
ATP6 & G9123A & silent & 2 & 8 \\
New mutation & & & & \\
ND6 & A14209G & N $\rightarrow$ D & 3 & 12 \\
ND5 & A12361G & T $\rightarrow$ A & 2 & 8 \\
ND5 & T14088C & silent & 3 & 12 \\
CO II & C8215T & silent & 3 & 12 \\
Cyt b & C15508T & silent & 2 & 8 \\
CO I & C6482T & silent & 2 & 8 \\
CO III & C9968T & silent & 2 & 8 \\
\hline
\end{tabular}

NT=nucleotide; AA, amino acid

2. mtDNA Mutations Found Only in CCA Patients

B

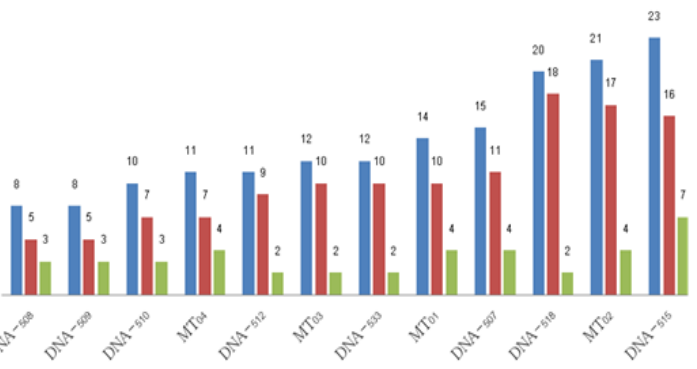

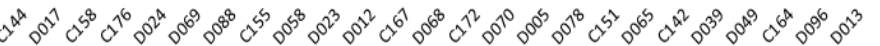

Figure 1. Number and Type of mtDNA Mutations Identified in Individual Subjects. A) Whole mtDNA sequence was determined in tumor tissues from 25 CCA patients. Similar mutations were found in tumor tissues and white blood cells from individual CCA patient. B) Whole mtDNA sequence was determined in white blood cells from 12 healthy subjects

2A

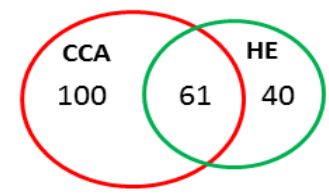

2B

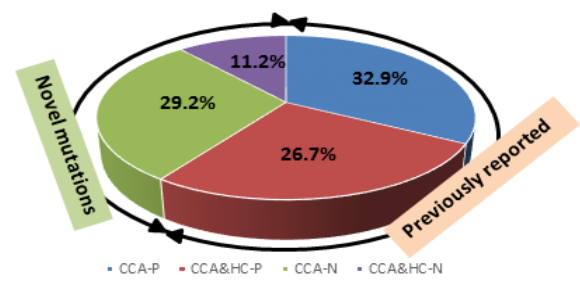

2C

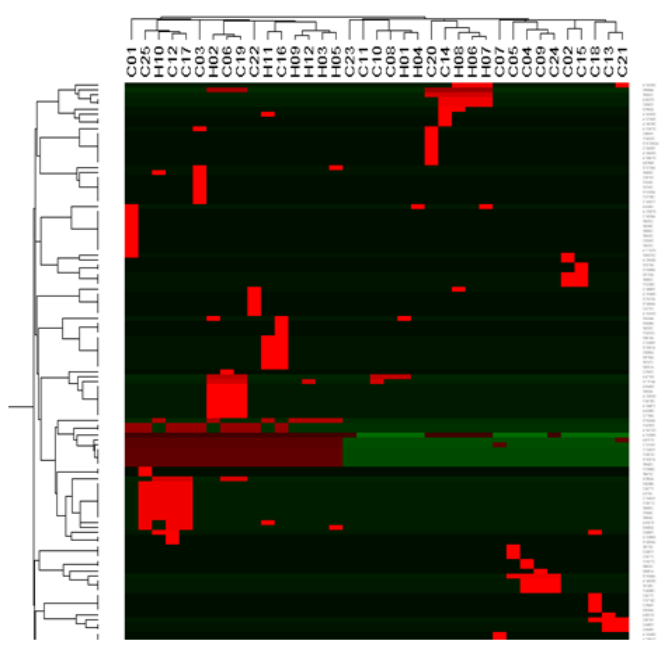

Figure 2. Cluster Analysis of mtDNA Mutations Found in CCA Patients and Healthy Persons. A) Mutations found both in CCA patients and healthy subjects (HE). B) Novel mutations were found in CCA patients. C) Total mtDNA mutations found analyzed using cluster analysis. Red square indicates the common mutations found in CCA patients and healthy subjects. red=mutation; green=normal

of which $43(27 \%)$ mutations were also found in healthy controls (Figure 2B). The mutations that were not matched to those reported in MITOMAP database were classified as new mutations. Based on this definition, there were $65(40 \%)$ determined, of which $47(29 \%)$ only in CCA patients (Table 2) and $18(11 \%)$ in healthy subjects. 


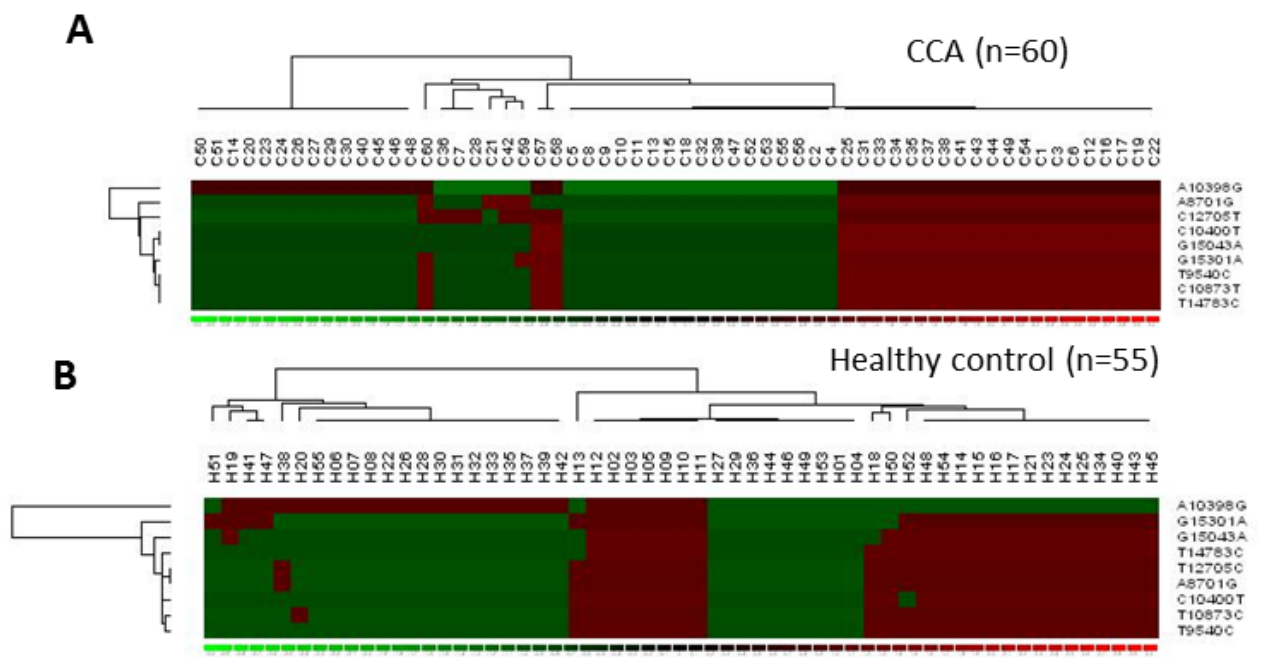

Figure 3. The Unsupervised Cluster Analysis for Association of mtDNA Mutations and CCA. The mtDNA mutations with high frequencies were analyzed in $60 \mathrm{CCA}$ and 55 healthy controls
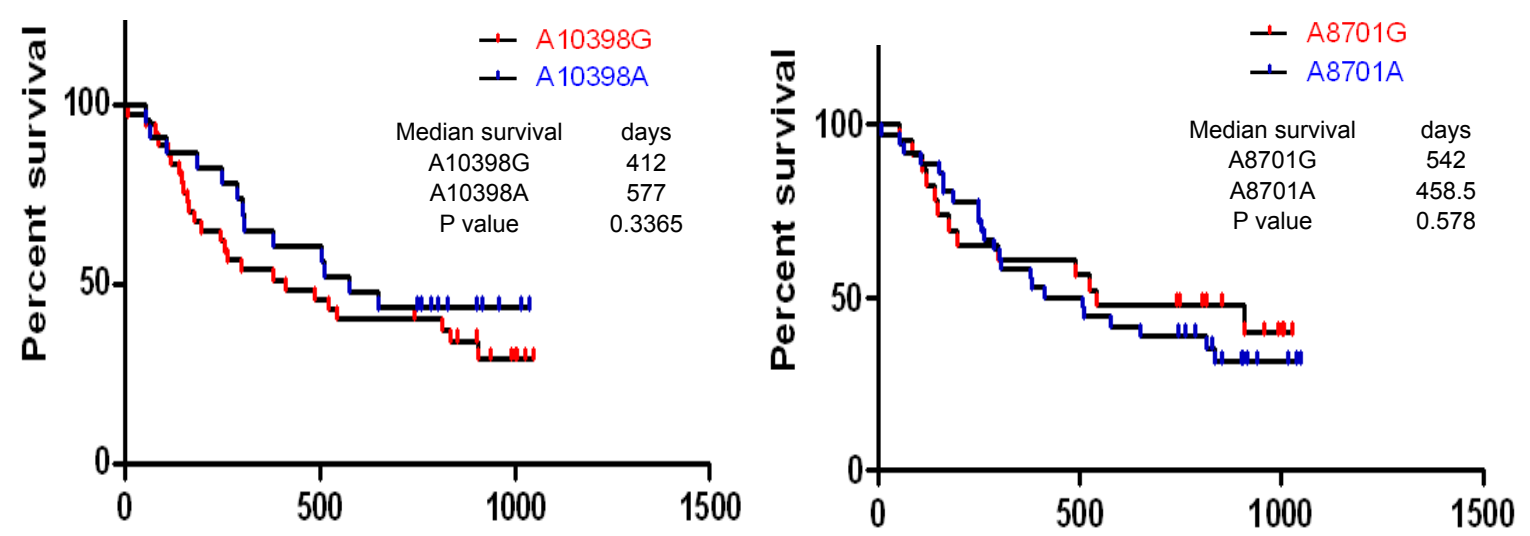

Figure 4. Survival Curves with Reference to mt DNA Mutations

High frequent mutations found in CCA patients were commonly found in healthy persons

Unsupervised cluster analysis of mtDNA mutations of $25 \mathrm{CCA}$ patients and 12 healthy persons is shown in Figure 2C. There was no unique mutation pattern which differentiated CCA patients from healthy persons. However, there were 9 mutations with high frequency observed in CCA subjects-A8701G $(\mathrm{A} \rightarrow \mathrm{T})$, T9540C, A10398G $(\mathrm{T} \rightarrow \mathrm{A}), \mathrm{C} 10400 \mathrm{~T}$; T10873C, C12705T, T14783C, G15043A and G15301A, respectively. To determine the association of these mtDNA mutations with CCA, these mutations were examined in white blood cells from a larger size (additional to the pilot set) to yield a total of 60 CCA cases and 55 healthy subjects. The unsupervised cluster analysis revealed these mutations in CCA were $36-46 \%$ and in healthy subjects were $43-49 \%$ (Figure 3).

\section{mtDNA mutations and clinical presentations of CCA}

To explore the association between mtDNA mutations and the clinical presentations of CCA patients, the number and position of mtDNA mutations found in individual CCA subjects were analyzed according to age, sex, histological type and metastasis status of the patients. Univariate analysis was determined using median of total mutation $(\leq 15 v s>15)$ and median of missense mutation $(\leq 4 v s>4)$ as cutting points. There were no significant correlations found between mtDNA mutations and clinical findings of CCA patients.

To examine whether the missense mutations (A10938G and $\mathrm{A} 8701 \mathrm{G}$ ) associated with the clinicopathology of disease and affected the patient survival, the survival analysis between patients who carried these mutations were analyzed versus those who had no those mutations. As shown in Figure 4, patients with mutation of A10938G exhibited trend of having shorter survival than those without mutation, however, this observation was not statistically significant.

\section{Discussion}

The observations of Otto Warburg on aerobic glycolysis or the "Warburg effect" has initiated and challenged the study of mitochondria in cancer etiology and carcinogenesis. In the present study, the whole mitochondrial genome from tumor tissues and corresponding white blood cells from a pilot set of 25 CCA patients revealed numerous mtDNA mutations in CCA patients, all of which were substitution of single point mutations and germline origins. The whole mitochondrial genomes from white blood cells of 12 healthy persons were compared to reveal any pathogenic mtDNA mutations associated with CCA. A subset of nine mutations, representing the most frequently observed in 
CCA group, were tested on the larger cohort involving 60 CCA patients and 55 controls. These mutations were observed in both groups with similar frequencies.

Cancer mtDNA mutations could either arise in the female germ line and predispose to cancer calledoncogenic germline mutation or arise in the mtDNA of the tissues and participate in the tumor progression process called-tumor-specific somatic mutations. Both germline and somatic mutations of mtDNA were reported for many cancers (reviewed in Chatterjee et al., 2006, Brandon et al., 2006). Many of those resulted in changing of amino acid (missense mutation) in the complex enzymes with predicted defect in their functions. In the current study, the whole mtDNA genome analysis revealed numerous new mtDNA mutations in tumor tissues from CCA patients, however, they were all germline mutations as they were also detected in the corresponding white blood cells.

There are three germline mtDNA mutation associated with cancer risks reported so far. The variant occurring in the ND3 gene (A10398G) was linked to increase risk for invasive breast cancer (Canter et al., 2005), while that on the D-Loop (T16189C) was associated with endometrial cancer (Liu et al., 2001), and the mtDNA COI gene variants were associated with prostate cancer (Tan et al., 2002; Herrmann et al., 2003; Krieg et al., 2004; Petros et al., 2005). To determine the significance of mtDNA mutations found in CCA patients, the whole mitochondrial genome sequences from CCA patients and those of white blood cells from the healthy controls were compared. Approximately one-third of mtDNA mutations found in CCA patients were also detected in the controls. Similar frequencies of the missense mutations were detected in both groups (36\% in CCA vs. $26.7 \%$ in the controls; $p=0.097)$. The nine most frequently observed mutations in the CCA group (32-44\%)-A8701G $(\mathrm{A} \rightarrow \mathrm{T})$, T9540C, A10398G $(\mathrm{T} \rightarrow \mathrm{A}), \mathrm{C} 10400 \mathrm{~T}, \mathrm{~T} 10873 \mathrm{C}, \mathrm{C} 12705 \mathrm{~T}$, T14783C, G15043A and G15301A-were determined in a larger joined cohort of total 60 CCA cases and 55 controls to determine the association of these mutations with CCA. As these mutations were also found in the controls with similar frequency, these mutations are probably the polymorphism of the Northeast Thais and may not associate with the disease.

In contrast to the present study, various somatic mutations ranging from 25 to $60 \%$ have been reported in mitochondrial coding regions in many cancers (Liu et al., 2003; Abu-Amero et al., 2005; Yin et al., 2010). The variant A10398G in the ND3 gene was reported as somatic mtDNA mutation in cancers of breast, colorectal and prostate (Canter et al., 2005; Zhu et al., 2005; AllalunisTurner et al., 2006), while the variant A8701G in the ATP6 gene was identified as somatic mtDNA mutation in the cancers of breast and thyroid (Tan et al., 2002; Abu-Amero et al., 2005). It should be noted here that these variants were also found in CCA patients but as germline mutation with $40 \%$ (24/60) frequency.

There were 65 new mutations revealed from the present study, of these $37 \%(24 / 65)$ were missense mutation and $63 \%(41 / 65)$ were silent mutations. The highest frequency of mtDNA mutations was found in Complex I. Complex I is the first site of the electron transport chain and active site
DOI:http://dx.doi.org/10.7314/APJCP.2015.16.5.1737 New Mitochondrial Mutations Found in Cholangiocarcinoma of reactive oxygen species production. The contribution of Complex I mutations to cancer largely due to the corresponding bioenergetics dysfunction that they cause. Mutations in mitochondrial genes encoding for Complex I have been linked to the development of several cancers (reviewed in Chatterjee et al., 2006).

To determine the influence of the mtDNA mutations in CCA, univariate analysis was performed in 25 CCA cases with sex, age, histological type and metastasis status of the patients. There was no significant correlation between the number of total mutations or missense mutations and clinical findings of the patients. This negative finding may be due to the small sample size. Likewise, the analysis should be done in a particular mutation rather than combined mutations as a whole.

In conclusion, the whole mitochondrial genome sequencing of CCA patient tissues and white blood cells were analyzed in comparison to those of white blood cells from healthy persons. There were 161 mutations found in CCA patients with 65 are new mutations. All mtDNA mutations found in CCA were substitution of point mutation with germline origin. Although, there is no direct association between mtDNA mutations and CCA observed in this study, it is the first mtDNA analysis reported in CCA. As the associations of mitochondria and cancer are collectively reported, it is of interest to explore the functions of the missense mutations reported in this study in a larger cohort. This may lead to the possibility of targeting mitochondria for cancer marker and therapy in the future.

\section{Acknowledgements}

This study was supported by Research grants from Khon Kaen University and the Higher Education Research Promotion and National Research University Project of Thailand, Office of the Higher Education Commission, through the Health cluster (SHeP-GMS) and the TRF Senior Research Scholar Grant to S. Wongkham, Thailand Research Fund (RTA5780012). We would like to thank Prof. Patcharee Lertrit, Faculty of Medicine, Siriraj hospital, Mahidol University, Thailand for her valuable criticism and Prof. Carlito B. Lebrilla, University of California Davis, USA for his comment and the English presentation of this manuscript.

\section{References}

Abu-Amero KK, Alzahrani AS, Zou M, Shi Y. (2005). High frequency of somatic mitochondrial DNA mutations in human thyroid carcinomas and complex I respiratory defect in thyroid cancer cell lines. Oncogene, 24, 1455-60.

Allalunis-Turner J, Ma I, Hanson J, Pearcey RG. (2006). mtDNA mutations in invasive cervix tumors: a retrospective analysis. Cancer lett, 243, 193-201.

Anderson S, Bankier AT, Barrell BG, et al. (1981). Sequence and organization of the human mitochondrial genome. Nature, 290, 457-65.

Bai Y, Attardi G. (1998). The mtDNA-encoded ND6 subunit of mitochondrial NADH dehydrogenase is essential for the assembly of the membrane arm and the respiratory function of the enzyme. EMBO J, 17, 4848-58. 


\section{Kanha Muisuk et al}

Brandon M, Baldi P, Wallace DC. (2006). Mitochondrial mutations in cancer. Oncogene, 25, 4647-62.

Canter JA, Kallianpur AR, Parl FF, Millikan RC. (2005). Mitochondrial DNA G10398A polymorphism and invasive breast cancer in African-American women. Cancer Res, 65, 8028-33.

Chatterjee A, Mambo E, Sidransky D. (2006). Mitochondrial DNA mutations in human cancer. Oncogene, 25, 4663-74.

DiMauro S, Schon EA. (2003). Mitochondrial respiratory-chain diseases. N Eng J Med, 348,2656-68.

Fogg VC, Lanning NJ, Mackeigan JP. (2011). Mitochondria in cancer: at the crossroads of life and death. Chin J Cancer, 30, 526-39.

Gaude E, Frezza C. (2014). Defects in mitochondrial metabolism and cancer. Cancer Met, 2, 10.

Herrmann PC, Gillespie JW, Charboneau L, et al. (2003). Mitochondrial proteome: altered cytochrome c oxidase subunit levels in prostate cancer. Proteomics, 3, 1801-10.

Holt IJ, Harding AE, Petty RK, Morgan-Hughes JA. (1990). A new mitochondrial disease associated with mitochondrial DNA heteroplasmy. Am J Hum Gen, 46, 428-33.

Huai JP, Ding J, Ye XH, Chen YP. (2014). Inflammatory bowel disease and risk of cholangiocarcinoma: evidence from a meta-analysis of population-based studies. Asian Pac J Cancer Prev, 15, 3477-82.

Hung WY, Wu CW, Yin PH, et al. (2010). Somatic mutations in mitochondrial genome and their potential roles in the progression of human gastric cancer. Biochi Biophys Acta, 1800, 264-70.

Khunluck T, Kukongviriyapan V, Puapairoj A, et al (2014). Association of NRF2 polymorphism with cholangiocarcinoma prognosis in Thai patients. Asian Pac J Cancer Prev, 15, 299-304.

Kim R, Emi M, Tanabe K. (2006). Role of mitochondria as the gardens of cell death. Cancer Chemo Pharmacol, 57, 545-53.

Krieg RC,Knuechel R, Schiffmann E, et al.(2004). Mitochondrial proteome: cancer-altered metabolism associated with cytochrome c oxidase subunit level variation. Proteomics, 4, 2789-95.

Liu VW, Shi HH, Cheung AN, et al. (2001). High incidence of somatic mitochondrial DNA mutations in human ovarian carcinomas. Cancer Res, 61, 5998-6001.

Liu VW, Yang HJ, Wang Y, et al. (2003). High frequency of mitochondrial genome instability in human endometrial carcinomas. Brit J Cancer, 89, 697-701.

Ma Y, Bai RK, Trieu R, Wong LJ. (2010). Mitochondrial dysfunction in human breast cancer cells and their 